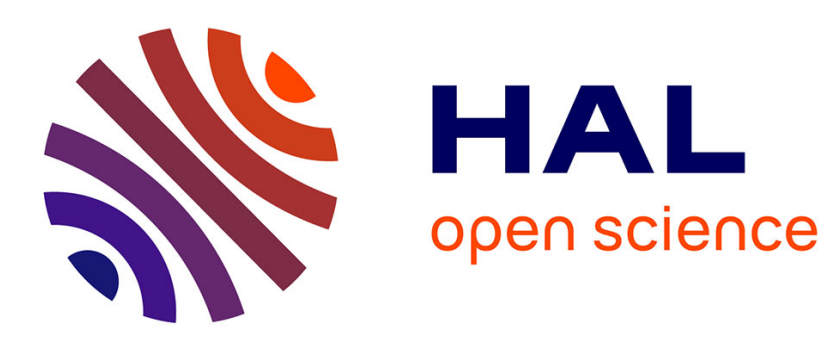

\title{
ON COMPACT ANISOTROPIC WEINGARTEN HYPERSURFACES IN EUCLIDEAN SPACE
}

\author{
Julien Roth, Abhitosh Upadhyay
}

\section{To cite this version:}

Julien Roth, Abhitosh Upadhyay. ON COMPACT ANISOTROPIC WEINGARTEN HYPERSURFACES IN EUCLIDEAN SPACE. Archiv der Mathematik, 2019, 113 (2), pp.213-224. 10.1007/s00013-019-01315-8 . hal-01985969

\section{HAL Id: hal-01985969 \\ https://hal.science/hal-01985969}

Submitted on 18 Jan 2019

HAL is a multi-disciplinary open access archive for the deposit and dissemination of scientific research documents, whether they are published or not. The documents may come from teaching and research institutions in France or abroad, or from public or private research centers.
L'archive ouverte pluridisciplinaire HAL, est destinée au dépôt et à la diffusion de documents scientifiques de niveau recherche, publiés ou non, émanant des établissements d'enseignement et de recherche français ou étrangers, des laboratoires publics ou privés. 


\title{
ON COMPACT ANISOTROPIC WEINGARTEN HYPERSURFACES IN EUCLIDEAN SPACE
}

\author{
JULIEN ROTH AND ABHITOSH UPADHYAY
}

\begin{abstract}
We show that, up to homotheties and translations, the Wulff shape $\mathcal{W}_{F}$ is the only compact embedded hypersurface of the Euclidean space satisfying $H_{r}^{F}=a H^{F}+b$ with $a \geqslant 0, b>0$, where $H^{F}$ and $H_{r}^{F}$ are respectively the anisotropic mean curvature and anisotropic $r$-th mean curvature associated with the function $F: \mathbb{S}^{n} \longrightarrow \mathbb{R}_{+}^{*}$. Further, we show that if the $L^{2}$-norm of $H_{r}^{F}-a H^{F}-b$ is sufficiently close to 0 then the hypersurface is close to the Wulff shape for the $W^{2,2}$-norm.
\end{abstract}

\section{INTRODUCTION}

The classical Alexandrov theorem [1] says that a closed embedded hypersurface of the Euclidean space must be a round sphere. The hypothesis, that the hypersurface should be embedded, is crucial as proved by the counter examples of Wente [15, Kapouleas 9] or Hsiang-Teng-Yu [7. Further, this result has been extended to scalar curvature and then higher order mean curvatures by Ros [11, 12 as well as for any concave function of the principal curvatures by Korevaar [10]. For higher order mean curvatures, the necessity of the embedding is still an open question.

Very recently, de Lima 2 proved a comparable result for the so called linear Weingarten hypersurfaces satisfying $H_{r}=a H+b$ for two real constants $a \geqslant 0$ and $b>0$, where $H$ and $H_{r}$ are respectively the mean curvature and the $r$-th mean curvature of the hypersurfaces. The hypersurfaces are supposed to be embedded in this result too.

In the present paper, we extend this result to higher order anisotropic mean curvatures and then, we study its stability.

Let $F: \mathbb{S}^{n} \longrightarrow \mathbb{R}_{+}^{*}$ be a smooth function satisfying the following convexity assumption

$$
A_{F}=\left(\nabla d F+F \operatorname{Id}_{\mid T_{x} \mathbb{S}^{n}}\right)_{x}>0,
$$

for all $x \in \mathbb{S}^{n}$, where $\nabla d F$ is the Hessian of $F$ and $>0$ means positive definite in the sense of quadratic forms. Now, we consider the following map

$$
\begin{aligned}
\phi: \mathbb{S}^{n} & \longrightarrow \mathbb{R}^{n+1} \\
x & \longmapsto F(x) x+\left(\operatorname{grad}_{\mid S^{n}} F\right)_{x} .
\end{aligned}
$$

The image $\mathcal{W}_{F}=\phi\left(\mathbb{S}^{n}\right)$ is called the Wulff shape of $F$ and is a smooth convex hypersurface of $\mathbb{R}^{n+1}$ due to condition (1). It is to note that if $F=1$, then the Wulff shape is the sphere $\mathbb{S}^{n}$.

Let $X:\left(M^{n}, g\right) \longrightarrow \mathbb{R}^{n+1}$ be an isometric immersion of $n$-dimensional closed,

2010 Mathematics Subject Classification. 53C42, 53A07, 49Q10.

Key words and phrases. Wulff shape, Weingerten hypersurfaces, Anisotropic mean curvature. 
connected and oriented Riemannian manifold $M$ into $\mathbb{R}^{n+1}$. We denote by $\nu$ a normal unit vector field globally defined on $M$, that is, we have $\nu: M \longrightarrow \mathbb{S}^{n}$. We set $S_{F}=-A_{F} \circ \mathrm{d} \nu$, where $A_{F}$ is defined in (1). The operator $S_{F}$ is called the $F$-Weingarten operator or anisotropic shape operator. In this anisotropic setting, we can define all the corresponding extrinsic quantities like anisotropic principal curvatures, anisotropic mean curvature and higher order mean curvatures (see the preliminaries section for the precise definitions).

All the above mentioned results by Alexandrov and Ros have analogues for anisotropic mean curvatures with the Wulff shape replacing the sphere (see [6]).

The first result of this paper is an isotropic version of the result of de Lima for linear Weingerten hypersurfaces. Namely, we have the following.

Theorem 1.1. Let $n \geqslant 2$ be an integer, $F: \mathbb{S}^{n} \longrightarrow \mathbb{R}_{+}^{*}$ a smooth function satisfying the convexity assumption (1) and let $M$ be a closed, connected and embedded hypersurface of $\mathbb{R}^{n+1}$. Assume that the higher order anisotropic mean curvature $H_{r}^{F}, r \in\{2, \cdots n\}$ never vanishes and satisfies $H_{r}^{F}=a H^{F}+b$ for some real constants $a \geqslant 0$ and $b>0$. Then, up to translations and homotheties, $M$ is the Wulff shape $\mathcal{W}_{F}$.

Here, a natural question arises related to the stability of the characterization in Theorem 1.1. We can ask, if $H_{r}^{F}$ is almost equal to $a H^{F}+b$ (in some sense to be precise), then the hypersurface will be close to the Wulff shape $\mathcal{W}_{F}$ or not?

In order to give an answer to this question, we introduce the following convenient notation. For three positive real numbers $r_{0}, h_{1}$ and $h_{2}$, we define $\mathcal{M}\left(r_{0}, h_{1}, h_{2}\right)$ as the set of all closed, connected and embedded hypersurface of $\mathbb{R}^{n+1}$ so that the extrinsic radius, that is, the radius of the smallest closed ball containing $M$, is smaller than $r_{0}$ whereas the first and second order anisotropic mean curvatures $H^{F}$ and $H_{2}^{F}$ satisfy

$$
\left\|H^{F}\right\|_{\infty} \leqslant h_{1} \quad \text { and } \quad \inf \left(H_{2}^{F}\right) \geqslant h_{2} .
$$

Now, we can prove the following result.

Theorem 1.2. Let $n \geqslant 2$ be an integer, $F: \mathbb{S}^{n} \longrightarrow \mathbb{R}_{+}^{*}$ a smooth function satisfying the convexity assumption (1) and let $M \in \mathcal{M}\left(r_{0}, h_{1}, h_{2}\right)$. Assume that the second order anisotropic mean curvature $H_{2}^{F}$ never vanishes and satisfies $H_{2}^{F}=a H^{F}+b+\varepsilon$ for some real constants $a \geqslant 0, b>0$ and $\varepsilon$ a smooth function. Set $\rho=\left(\frac{V(M)}{V\left(\mathcal{W}_{F}\right)}\right)^{\frac{1}{n}}$. Then there exists a smooth parametrisation $\psi: \mathcal{W}_{\rho F} \longrightarrow M$, a vector $c_{0} \in \mathbb{R}^{n+1}$ and an explicit constant $K$ depending on $n, F, r_{0}, h_{1}$ and $h_{2}$ so that

$$
\left\|\psi-\mathrm{Id}-c_{0}\right\|_{W^{2,2}\left(\mathcal{W}_{\rho F}\right)} \leqslant K\|\varepsilon\|_{2} \text {. }
$$

Here $\mathcal{W}_{\rho F}=\rho \mathcal{W}_{F}$ is just the image of $\mathcal{W}_{F}$ by the homothety of center 0 and ratio $\rho$.

This result is an improvement of the result obtained by the first author in 13 . and [14] for almost constant mean curvature and scalar curvature in the $L^{2}$-sense. In particular, if $F$ is constant, we get the following corollary for the anisotropic case.

Corollary 1.3. Let $n \geqslant 2$ be an integer and assume that $M \in \mathcal{M}\left(r_{0}, h_{1}, h_{2}\right)$. Assume that the second order anisotropic mean curvature $\mathrm{H}_{2}$ never vanishes and satisfies $\mathrm{H}_{2}=a H+b+\varepsilon$ for some constants $a \geqslant 0, b>0$ and $\varepsilon$ a smooth function. 
Set $\rho=\left(\frac{V(M)}{V\left(\mathbb{S}^{n}\right)}\right)^{\frac{1}{n}}$. Then there exist a smooth parametrisation $\psi: \mathbb{S}^{n}(\rho) \longrightarrow M, a$ vector $c_{0} \in \mathbb{R}^{n+1}$ and a constant $K^{\prime}$ depending on $n, r_{0}, h_{1}$ and $h_{2}$ so that

$$
\left\|\psi-\mathrm{Id}-c_{0}\right\|_{W^{2,2}\left(\mathbb{S}^{n}(\rho)\right)} \leqslant K^{\prime}\|\varepsilon\|_{2} \text {. }
$$

This result has also to be compared with the results of [13] and [14]. In particular for $a=0$, this gives a stability result associated with the Alexandrov theorem for $\mathrm{H}_{2}$.

\section{Preliminaries}

Here, we recall the basics of anisotropic mean curvatures. These facts are classical, hence, we will not write their proofs. First, let $F: \mathbb{S}^{n} \longrightarrow \mathbb{R}_{+}^{*}$ be a smooth function satisfying the following convexity assumption (1)

$$
A_{F}=\left(\nabla d F+F \operatorname{Id}_{T_{x} \mathbb{S}^{n}}\right)_{x}>0
$$

at any point $x \in \mathbb{S}^{n}$, in the sense of quadratic forms and where $\nabla d F$ is the Hessian of $F$. Now, we consider the following map

$$
\begin{aligned}
\phi: \mathbb{S}^{n} & \longrightarrow \mathbb{R}^{n+1} \\
x & \longmapsto F(x) x+\left(\operatorname{grad}_{\mathbb{S}^{n}} F\right)_{x} .
\end{aligned}
$$

The image $\mathcal{W}_{F}=\phi\left(\mathbb{S}^{n}\right)$, which is also a smooth hypersurface of $\mathbb{R}^{n+1}$, is called the Wulff shape of $F$. Moreover, from the convexity condition $(1), \mathcal{W}_{F}$ is convex. Note that if $F$ is a positive constant $c$, the Wulff shape $\mathcal{W}_{F}$ is just the sphere of radius $c$.

Let $\left(M^{n}, g\right)$ be a closed, connected, oriented Riemannian manifold isometrically immersed into $\mathbb{R}^{n+1}$ by $X$ and denote by $\nu$ its Gauss map. The (real-valued) second fundamental form $B$ of the immersion is defined by

$$
B(Y, Z)=\left\langle\bar{\nabla}_{Y} \nu, Z\right\rangle
$$

for any $Y, Z \in \Gamma(T M)$, where $\langle\cdot, \cdot\rangle$ and $\bar{\nabla}$ are respectively the Riemannian metric and the Riemannian connection of $\mathbb{R}^{n+1}$. We also denote by $S$ the Weingarten operator, which is the $(1,1)$-tensor associated with $B$ via the metric $g$.

We consider $N_{F}=\phi(\nu): M \longrightarrow \mathcal{W}_{F}$, the anisotropic Gauss map of $M$. We set $S_{F}=-d N_{F}=-A_{F} \circ d \nu=A_{F} \circ S$ which is the anisotropic shape operator, also called $F$-Weingarten operator and its eigenvalues $\kappa_{1}, \cdots, \kappa_{n}$ are the anisotropic principal curvatures. Now let us recall that the anisotropic higher order mean curvatures $H_{r}^{F}$ are defined by

$$
H_{r}^{F}=\frac{1}{\left(\begin{array}{l}
n \\
r
\end{array}\right)} \sigma_{r}\left(S_{F}\right),
$$

where $\sigma_{r}\left(S_{F}\right)$ is the $r$-th elementary symmetric polyniomial with $n$ variables computed for anisotropic principal cruvatures $\kappa_{1}, \cdots, \kappa_{n}$.

We denote simply by $H^{F}$ the anisotropic mean curvature $H_{1}^{F}$. Moreover, for convenience, we set $H_{0}^{F}=1$ and $H_{n+1}^{F}=0$ by convention. For the Wulff shape $\mathcal{W}_{F}$, $\kappa_{1}=\kappa_{2}=\cdots=\kappa_{n}$ are nonzero constants. Moreover, if $\kappa_{1}=\kappa_{2}=\cdots=\kappa_{n}$, then the hypersurface has to be the Wulff shape (up to homotheties and translations).

Lemma 2.1. Let $r \in\{1, \cdots n-1\}$. If $H_{r+1}>0$ then

(1) $H_{j}^{F}>0$ for all $j \in\{1, \cdots, r\}$, 
(2) $\left(H_{r}^{F}\right)^{\frac{1}{r}} \leqslant\left(H_{r-1}^{F}\right)^{\frac{1}{r-1}} \leqslant \cdots \leqslant\left(H_{2}^{F}\right)^{\frac{1}{2}} \leqslant H^{F}$. Moreover, in any of these inequalities, equality occurs at a point $p$ if and only if all the anisotropic principal curvatures at $p$ are equal. Hence, equality occurs everywhere if and only if $M$ is the Wulff shape $\mathcal{W}_{F}$, up to translations and homotheties.

We want to point out that the error term in the inequality $\left(H_{2}^{F}\right)^{\frac{1}{2}} \leqslant H^{F}$ is well known and easy to compute since we have

$$
n(n-1)\left(\left(H^{F}\right)^{2}-H_{2}^{F}\right)=\left\|\tau_{F}\right\|^{2},
$$

where $\tau_{F}=S_{F}-H^{F} \mathrm{Id}$ is the anisotropic umbilicity tensor.

We also recall the anisotropic analogue of the classical Hsiung-Minkowski formulas [8]. The proof can be found in [5] for instance.

Lemma 2.2. Let $r \in\{0, \cdots n-1\}$. Then, we have

$$
\int_{M}\left(F(\nu) H_{r}^{F}+H_{r+1}^{F}\langle X, \nu\rangle\right) d v_{g}=0 .
$$

Note that for the particular case $r=0$, we get the following

$$
\int_{M} H^{F}\langle X, \nu\rangle d v_{g}=-\int_{M} F(\nu) d v_{g} .
$$

For more convenience, we will denote $V_{F}(M)=\int_{M} F(\nu) d v_{g}$.

Finally, we recall these two useful results in the case of hypersurfaces which are embedded and so, bound some domain in $\mathbb{R}^{n+1}$. First, we have this classical identity obtained directly by the divergence formula

$$
\int_{M}\langle X, \nu\rangle d v_{g}=-(n+1) V(\Omega)
$$

where $V(\Omega)$ is the volume of the domain $\Omega$ bounded by $M$. Secondly, we have the anisotropic analogue of the well-known Heintze-Karcher inequality.

Lemma 2.3 ([5]). If $M$ is embedded (so bounds a domain $\Omega$ ) and $H^{F}$ is everywehere positive, then the following inequality holds

$$
\int_{M} \frac{F(\nu)}{H^{F}} d v_{g} \geqslant(n+1) V(\Omega),
$$

with equality if and only if $M$ is the Wulff shape $\mathcal{W}_{F}$ (up to translations and homotheties).

Finally, we add that we use the following convention for the $L^{p}$-norms. For a smooth function $f$ defined on $M$, the $L^{p}$-norm of $f$ is defined by

$$
\|f\|_{p}=\left(\frac{\int_{M}|f|^{p} d v_{g}}{V(M)}\right)^{\frac{1}{p}} .
$$




\section{Proof of Theorem 1.1}

Since $M$ is compact and embedded, it bounds a domain in $\mathbb{R}^{n+1}$. We denote this domain by $\Omega$. Moreover, always by compactness, $M$ has at least an elliptic point. Since $H_{r}^{F}$ never vanishes, we can assume by connectedness and the positivity of $A_{F}$ that $H_{r}^{F}$ is positive everywhere (by choosing $\nu$ as the inner normal unit vector). Now, from the anisotropic Hsiung-Minkowski formula, we have

$$
\begin{aligned}
0 & =\int_{M}\left(H_{r-1}^{F} F(\nu)+H_{r}^{F}\langle X, \nu\rangle\right) d v_{g} \\
& =\int_{M}\left(H_{r-1}^{F} F(\nu)+a H^{F}\langle X, \nu\rangle+b\langle X, \nu\rangle\right) d v_{g},
\end{aligned}
$$

by the assumption $H_{r}^{F}=a H^{F}+b$. Moreover, by the Hsiung-Minkowski formula again for $H^{F}$ and the divergence theorem, we get that

$$
\int_{M} H^{F}\langle X, \nu\rangle d v_{g}=-V_{F}(M), \quad \text { and } \quad \int_{M}\langle X, \nu\rangle d v_{g}=-(n+1) V(\Omega),
$$

which gives after substitution in (5)

$$
\int_{M} H_{r-1}^{F} F(\nu) d v_{g}-a V_{F}(M)-b(n+1) V(\Omega)=0 .
$$

Since $H_{r}^{F}$ is positive everywhere, by Lemma 2.1. $H_{r-1}^{F}$ is also positive everywhere and $\left(H_{r-1}^{F}\right)^{\frac{1}{r-1}} \geqslant\left(H_{r}^{F}\right)^{\frac{1}{r}}$ with equality if and only if the point is anisotropically umbilical. Hence, we get

$$
a V_{F}(M)+b(n+1) V(\Omega) \geqslant \int_{M} F(\nu)\left(H_{r}^{F}\right)^{\frac{r-1}{r}} d v_{g}
$$

Writing $\left(H_{r}^{F}\right)^{\frac{r-1}{r}}=H_{r}^{F}\left(H_{r}^{F}\right)^{\frac{-1}{r}}$ and using $H_{r}^{F}=a H^{F}+b$ again, we obtain

$$
a V_{F}(M)+b(n+1) V(\Omega) \geqslant a \int_{M} F(\nu) H^{F}\left(H_{r}^{F}\right)^{-\frac{1}{r}} d v_{g}+b \int_{M} F(\nu)\left(H_{r}^{F}\right)^{-\frac{1}{r}} d v_{g} .
$$

Since $a, b$ are nonnegative numbers and $F$ is a positive function, we get, from $\left(H_{r}^{F}\right)^{\frac{1}{r}} \leqslant H^{F}$, the following

$$
a V_{F}(M)+b(n+1) V(\Omega) \geqslant a V_{F}(M)+b \int_{M} \frac{F(\nu)}{H^{F}} d v_{g} .
$$

Finally, we use the anisotropic version of the Heintze-Karcher inequality

$$
\int_{M} \frac{F(\nu)}{H^{F}} d v_{g} \geqslant(n+1) V(\Omega)
$$

to get

$$
a V_{F}(M)+b(n+1) V(\Omega) \geqslant a V_{F}(M)+b(n+1) V(\Omega),
$$

which means that all the previous inequalities are in fact equalities. In particular, we have $\left(H_{r-1}^{F}\right)^{\frac{1}{r-1}}=\left(H_{r}^{F}\right)^{\frac{1}{r}}$ at every point and so $M$ is totally anisotropically umblical. Hence, up to homotheties and translations, $M$ is the Wulff shape $\mathcal{W}_{F}$. This concludes the proof. 


\section{Proof of Theorem 1.2}

For the sake of clarity, we first proof the following lemma.

Lemma 4.1. Let $n \geqslant 2$ be an integer, $F: \mathbb{S}^{n} \longrightarrow \mathbb{R}_{+}^{*}$ be a smooth function satisfying the convexity assumption (1) and let $M$ be a closed connected and embedded hypersurface of $\mathbb{R}^{n+1}$. Assume that the second order anisotropic mean curvature $H_{2}^{F}$ never vanishes and satisfies $H_{2}^{F}=a H^{F}+b+\varepsilon$ for some real constants $a \geqslant 0$, $b>0$ and $\varepsilon$ a smooth function. Then we have

$$
\left\|\tau_{F}\right\|_{2}^{2} \leqslant 2 n(n-1)\left(\frac{\sup (F(\nu))}{\inf \left(\left(H_{2}^{F}\right)\right)^{\frac{1}{2}}}+R\right) \frac{\left\|H^{F}\right\|_{\infty}}{\inf (F(\nu))}\|\varepsilon\|_{2}^{2}
$$

where $R$ is the extrinsic radius of $M$.

Proof: By the Hsiung-Minkowski formula for $H_{2}^{F}$ and $H_{F}$

$$
\int_{M}\left(F(\nu) H_{1}^{F}+\langle X, \nu\rangle\right) d v_{g}=0
$$

and using the assumption $H_{2}^{F}=a H^{F}+b+\varepsilon$, we have

$$
\begin{aligned}
0 & =\int_{M}\left(F(\nu) H^{F}+a H^{F}\langle X, \nu\rangle+b\langle X, \nu\rangle+\varepsilon\langle X, \nu\rangle\right) d v_{g} \\
& =\int_{M} F(\nu) H^{F} d v_{g}-a V_{F}(M)-b(n+1) V(\Omega)+\int_{M} \varepsilon\langle X, \nu\rangle d v_{g},
\end{aligned}
$$

where we have used both relations (3) and (4).

Now, from (2), we have

$$
H^{F}=\left(H_{2}^{F}\right)^{\frac{1}{2}}+\frac{\left\|\tau_{F}\right\|^{2}}{n(n-1)\left(H^{F}+\left(H_{2}^{F}\right)^{\frac{1}{2}}\right)} .
$$

Using this in (8) gives

$$
\begin{aligned}
0= & \int_{M}\left(H_{2}^{F}\right)^{\frac{1}{2}} F(\nu) d v_{g}+\frac{1}{n(n-1)} \int_{M} \frac{F(\nu)\left\|\tau_{F}\right\|^{2}}{H^{F}+\left(H_{2}^{F}\right)^{\frac{1}{2}}} d v_{g} \\
& -a V_{F}(M)-b(n+1) V(\Omega)+\int_{M} \varepsilon\langle X, \nu\rangle d v_{g} .
\end{aligned}
$$

Now, we consider the first term of the right hand side. We have

$$
\begin{aligned}
\int_{M}\left(H_{2}^{F}\right)^{\frac{1}{2}} F(\nu) d v_{g}= & \int_{M} H_{2}^{F}\left(H_{2}^{F}\right)^{-\frac{1}{2}} F(\nu) d v_{g} \\
= & a \int_{M} F(\nu) H^{F}\left(H_{2}^{F}\right)^{-\frac{1}{2}} d v_{g}+b \int_{M} F(\nu)\left(H_{2}^{F}\right)^{-\frac{1}{2}} d v_{g} \\
& +\int_{M} \varepsilon F(\nu)\left(H_{2}^{F}\right)^{-\frac{1}{2}} d v_{g} \\
\geqslant & a V_{F}(M)+b \int_{M} \frac{F(\nu)}{H^{F}} d v_{g}+\int_{M} \varepsilon F(\nu)\left(H_{2}^{F}\right)^{-\frac{1}{2}} d v_{g} \\
\geqslant & a V_{F}(M)+b(n+1) V(\Omega)+\int_{M} \varepsilon F(\nu)\left(H_{2}^{F}\right)^{-\frac{1}{2}} d v_{g}
\end{aligned}
$$


where we have used successively the assumption $H_{2}^{F}=a H^{F}+b+\varepsilon$, the fact that $\left(H_{2}^{F}\right)^{-\frac{1}{2}} \geqslant \frac{1}{H^{F}}$ and the Heintze-Karcher inequality.

Thus, from (9) and (10), we get

$$
\begin{aligned}
\frac{1}{n(n-1)} \int_{M} \frac{F(\nu)\left\|\tau_{F}\right\|^{2}}{H^{F}+\left(H_{2}^{F}\right)^{\frac{1}{2}}} d v_{g} & \leqslant-\int_{M} \varepsilon F(\nu)\left(H_{2}^{F}\right)^{-\frac{1}{2}} d v_{g}-\int_{M} \varepsilon\langle X, \nu\rangle d v_{g} \\
& \leqslant\left(\frac{\sup (F(\nu))}{\inf \left(\left(H_{2}^{F}\right)\right)^{\frac{1}{2}}}+R\right) V(M)\|\varepsilon\|_{2}^{2}
\end{aligned}
$$

where $R$ is the extrinsic radius of $M$, that is, the radius of the smallest closed ball containing $M$.

Moreover, using again the fact that $\left(H_{2}^{F}\right)^{\frac{1}{2}} \leqslant H^{F}$, we obtain the following

$$
\left\|\tau_{F}\right\|_{2}^{2} \leqslant 2 n(n-1)\left(\frac{\sup (F(\nu))}{\inf \left(\left(H_{2}^{F}\right)\right)^{\frac{1}{2}}}+R\right) \frac{\left\|H^{F}\right\|_{\infty}}{\inf (F(\nu))}\|\varepsilon\|_{2}^{2},
$$

which achieves the proof of the lemma.

Now, we will combine Lemma 4.1 with the following result of de Rosa and Gioffrè to prove Theorem 1.2 .

Theorem 4.2 (De Rosa-Gioffrè [3, 4]). Let $n>2, p \in(1,+\infty)$ and $F: \mathbb{S}^{n} \longrightarrow \mathbb{R}_{+}^{*}$ satisfying the convexity assumption (1). There exist a constant $\delta_{0}=\delta_{0}(n, p, F)>0$ such that if $M$ is closed hypersurface into $\mathbb{R}^{n+1}$ satisfying

$$
\operatorname{Vol}(M)=V\left(\mathcal{W}_{F}\right) \quad \text { and } \quad \int_{M}\left\|\tau_{F}\right\|^{p} d v_{g} \leqslant \delta_{0}
$$

then there exist a smooth parametrisation $\psi: \mathcal{W}_{F} \longrightarrow M$, a vector $c_{0} \in \mathbb{R}^{n+1}$ and a constant $C$ depending on $n, p$ and $F$ so that

$$
\left\|\psi-\mathrm{Id}-c_{0}\right\|_{W^{2, p}\left(\mathcal{W}_{F}\right)} \leqslant C\left\|\tau_{F}\right\|_{p} .
$$

Moreover, if $p \in(1, n]$, then the condition $\int_{M}\left\|\tau_{F}\right\|^{p} d v_{g} \leqslant \delta_{0}$ can be dropped.

Here, it is important to mention that the volume of $M$ is supposed to be equal to $V\left(\mathcal{W}_{F}\right)$. If we do not assume this, the same holds replacing $\mathcal{W}_{F}$ by the homothetic of $\mathcal{W}_{F}$ of volume equal to $V(M)$, that is for $\mathcal{W}_{\rho F}$ for $\rho=\left(\frac{V(M)}{V\left(\mathcal{W}_{F}\right)}\right)^{\frac{1}{n}}$. The authors first prove this result for convex hypersurfaces in [3] and then explain how to remove convexity in [4].

\section{One last Result}

We finish this paper by proving a final result in the same spirit of Theorem 1.2 . It is well known that a closed connected immersed hypersurface of the Euclidean space, with constant mean curvature, is a round sphere if the scalar product $\langle X, \nu\rangle$ between the position vector and the normal vector has fixed sign. Of course, the hypersurface is not supposed to be embedded because in this case, the assumption $\langle X, \nu\rangle$ has fixed sign is superfluous. An anisotropic version of this result has also been proved in [5]. We prove stability result for this characterization. Before stating the result, we introduce $\mathcal{M}^{\prime}\left(r_{0}, r_{1}, h_{1}\right)$ as the set of all closed, connected and 
embedded hypersurface of $\mathbb{R}^{n+1}$ so that the support function satisfies inf $|\langle X, \nu\rangle| \geqslant$ $r_{0}$, the extrinsic radius is smaller than $r_{1}$ and the $L^{\infty}$ norm of the anisotropic mean curvature is smaller than $h_{1}$. Namely, we have:

Theorem 5.1. Let $n \geqslant 2$ be an integer, $F: \mathbb{S}^{n} \longrightarrow \mathbb{R}_{+}^{*}$ a smooth function satisfying the convexity assumption (1) and let $M \in \mathcal{M}^{\prime}\left(r_{0}, r_{1}, h_{1}\right)$. Assume that the support function $\langle X, \nu\rangle$ has fixed sign and there exist a non-zero constant $h_{0}$ and a smooth function $\varepsilon$ so that $H^{F}=h_{0}+\varepsilon$. Set $\rho=\left(\frac{V(M)}{V\left(\mathcal{W}_{F}\right)}\right)^{\frac{1}{n}}$. Then there exist a smooth parametrisation $\psi: \mathcal{W}_{\rho F} \longrightarrow M$, a vector $c_{0} \in \mathbb{R}^{n+1}$ and an explicit constant $K_{1}$ depending on $n, r_{0}, r_{1}, h_{1}, F$ so that

$$
\left\|\psi-\mathrm{Id}-c_{0}\right\|_{W^{2,2}\left(\mathcal{W}_{\rho F}\right)} \leqslant K_{1}\|\varepsilon\|_{1}^{\frac{1}{2}} .
$$

Moreover, if $\|\varepsilon\|_{\infty} \leqslant \alpha h_{0}$ for some $\alpha \in(0,1)$, then, there exist another explicit constant $K_{2}$ depending on $n, r_{0}, F$ so that

$$
\left\|\psi-\mathrm{Id}-c_{0}\right\|_{W^{2,2}\left(\mathcal{W}_{\rho F}\right)} \leqslant K_{2}\|\varepsilon\|_{\infty}^{\frac{1}{2}} .
$$

Remark 5.2. This last theorem says that if the support function has constant sign, and $H^{F}$ is almost constant, then the hypersurface is $W^{2,2}$-close to the Wulff shape and either the $L^{1}$ or $L^{\infty}$-norm of the error term $\varepsilon$ quantify this closeness. But for a control by the $L^{1}$-norm of the error, the counterpart is that the constant $K_{1}$ depends on more geometric quantities as for a control by the $L^{\infty}$-norm.

Proof: First, we compute

$$
\begin{aligned}
\int_{M}\langle X, \nu\rangle\left(\left(H^{F}\right)^{2}-H_{2}^{F}\right) d v_{g} & =h_{0} \int_{M} H^{F}\langle X, \nu\rangle d v_{g}+\int_{M} \varepsilon H^{F}\langle X, \nu\rangle d v_{g}-\int_{M} H_{2}^{F}\langle X, \nu\rangle d v_{g} \\
& =-h_{0} V_{F}(M)+\int_{M} F(\nu) H^{F} d v_{g}+\int_{M} \varepsilon H^{F}\langle X, \nu\rangle d v_{g}
\end{aligned}
$$

where we have used the first and second Hsiung-Minkowski formulas. Replacing $H^{F}$ by $h_{0}+\varepsilon$ in the second term of the right hand side, we get

$$
\begin{aligned}
\int_{M}\langle X, \nu\rangle\left(\left(H^{F}\right)^{2}-H_{2}^{F}\right) d v_{g} & =-h_{0} V_{F}(M)+h_{0} V_{F}(M)+\int_{M} \varepsilon F(\nu) d v_{g}+\int_{M} \varepsilon H^{F}\langle X, \nu\rangle d v_{g} \\
& =\int_{M} \varepsilon F(\nu) d v_{g}+\int_{M} \varepsilon H^{F}\langle X, \nu\rangle d v_{g} .
\end{aligned}
$$

Since $\left(H^{F}\right)^{2}-H_{2}^{F}=\frac{1}{n(n-1)}\left\|\tau_{F}\right\|^{2}$ and from the assumptions that $\langle X, \nu\rangle$ has fixed sign and $\inf |\langle X, \nu\rangle| \geqslant r_{0}$, we obtain

$$
\frac{r_{0}}{n(n-1)} \int_{M}\left\|\tau_{F}\right\|^{2} d v_{g} \leqslant \int_{M} \varepsilon F(\nu) d v_{g}+\int_{M} \varepsilon H^{F}\langle X, \nu\rangle d v_{g} .
$$

First, we deduce

$$
\left\|\tau_{F}\right\|_{2}^{2} \leqslant \frac{n(n-1)}{r_{0}}\left(\sup F(\nu)+R\left\|H^{F}\right\|_{\infty}\right)\|\varepsilon\|_{1},
$$

where we have used that $|\langle X, \nu\rangle| \leqslant R$. Combining (14) with the result of de Rosa and Gioffrè, we get the first point of the theorem by setting

$$
K_{1}=C\left[\frac{n(n-1)}{r_{0}}\left(\sup F(\nu)+R\left\|H^{F}\right\|_{\infty}\right)\right]^{\frac{1}{2}},
$$


where $C$ is the constant of de Rosa and Gioffrè's result which depends only on $n$ and $F$, since here $p=2$. Second, from 13 , we get

$$
\begin{aligned}
\left\|\tau_{F}\right\|_{2}^{2} V(M) & \leqslant \frac{n(n-1)}{r_{0}}\left(\int_{M} \varepsilon F(\nu) d v_{g}+\int_{M} \varepsilon H^{F}\langle X, \nu\rangle d v_{g}\right) \\
& \leqslant \frac{n(n-1)}{r_{0}}\left(\sup F(\nu) V(M)+\int_{M}\left|H^{F}\langle X, \nu\rangle\right| d v_{g}\right)\|\varepsilon\|_{\infty} .
\end{aligned}
$$

Moreover, if there exist $\alpha \in(0,1)$ so that $\|\varepsilon\|_{\infty} \leqslant \alpha h_{0}$, there $\left|H_{F}\right| \geqslant(1-\alpha)\left|h_{0}\right|>0$. Hence, since both $H^{F}$ and $\langle X, \nu\rangle$ have fixed sign, we have

$$
\int_{M}\left|H^{F}\langle X, \nu\rangle\right| d v_{g}=\left|\int_{M} H^{F}\langle X, \nu\rangle d v_{g}\right|=\int_{M} F(\nu) d v_{g}
$$

by the first Hsiung-Minkowski formula.

From this and $(15)$, we obtain

$$
\left\|\tau_{F}\right\|_{2}^{2} \leqslant \frac{2 n(n-1) \sup F(\nu)}{r_{0}}\|\varepsilon\|_{\infty}
$$

and we conclude again by the result of de Rosa and Gioffrè by setting

$$
K_{2}=C\left[\frac{2 n(n-1) \sup F(\nu)}{r_{0}}\right] \text {. }
$$

Note that $K_{2}$ depends only on $n, F$ and $r_{0}$.

\section{Acknowledgements}

Second author is supported by National Post-doctoral fellowship of Science and Engineering Research Board (File no. PDF/2017/001165), India. He would also like to express his thanks to Professor Patrice Philippon (DR CNRS, resp. LIA IFPM) for providing necessary support to stay in France and the Laboratoire d'Analyse et de Mathématiques Appliquées, Marne-la-Vallée, for local hospitality during the preparation of this paper.

\section{REFERENCES}

[1] A.D. Alexandrov, A characteristic property of spheres, Ann. Mat. Pura Appl., 58 (1962), 303-315.

[2] E. De Lima, A note on compact Weingarten hypersurfaces embedded in $\mathbb{R}^{n+1}$, Arch. Math. (Basel), 111, (2018), 669-672.

[3] A. De Rosa \& S. Gioffrè, Quantitative stability for anisotropic nearly umbilical hypersurfaces, J. Geom. Anal., in press.

[4] A. De Rosa \& S. Gioffrè, Absence of bubbling phenomena for non convex anisotropic nearly umbilical and quasi Einstein hypersurfaces, arXiv: 1803.09118

[5] Y. He \& H. Li, Integral formula of Minkowski type and new characterization of the Wulff shape, Acta Math. Sinica, 24(4) (2008), 697-704.

[6] Y. He, H. Li, H. Ma \& J. Ge, Compact embedded hypersurfaces with constant higher order anisotropic mean curvature, Indiana Univ. Math. J. 58 (2009), 853-868.

[7] W. Hsiang, Z. Teng and W. Yu, New examples of constant mean curvature immersions of (2k-1)-spheres into Euclidean 2k-space, Ann. Math., 117(3) (1983), 609-625 .

[8] C.C. Hsiung, Some integral formulas for closed hypersurfaces, Math. Scand. 2 (1954), 286294.

[9] N. Kapouleas, Constant mean curvature surfaces constructed by fusing Wente tori, Invent. Math., 119(3), (1995), 443-518.

[10] N. J. Korevaar, Sphere theorems via Alexandrov constant Weingarten curvature hypersurfaces: appendix to a note of A. Ros, J. Differential Geom. 27 (1988), 221-223. 
[11] A. Ros, Compact hypersurfaces with constant scalar curvature and a congruence theorem J. Differential Geom. 27(2) (1988), 215-223.

[12] A. Ros, Compact hypersurfaces with constant higher order mean curvatures, Rev. Mat. Iberoamericana 3(3-4) (1987), 447-453.

[13] J. Roth, Rigidity results for geodesic spheres in space forms, Differential Geometry, Proceedings of the VIII International Colloquium, (World Scientific, 2009), 156-163.

[14] J. Roth, New stability results for spheres and Wulff shapes, Comm. in Math. in press.

[15] H. Wente, Counterexample to a conjecture of H. Hopf, Pac. J. Math., 121(1) (1986), 193-243.

(J. ROTH) Laboratoire D'Analyse et de Mathématiques Appliquées, Upem-UpeC, CNRS, F-77454 MARNE-LA-VALLÉE

E-mail address: julien.roth@u-pem.fr

(A. Upadhyay) Indian Institute of Science, Department of Mathematics, BangaLORE, 560012, INDIA

E-mail address: abhi.basti.ipu@gmail.com 